\title{
Stepping Motion for a Human-like Character to Maintain Balance against Large Perturbations
}

\author{
Shunsuke Kudoh \\ University of Tokyo \\ Tokyo, Japan \\ Email: kudoh@cvl.iis.u-tokyo.ac.jp
}

\author{
Taku Komura \\ City University of Hong Kong \\ Hong Kong, China \\ Email: taku@ieee.org
}

\author{
Katsushi Ikeuchi \\ University of Tokyo \\ Tokyo, Japan \\ Email: ki@cvl.iis.u-tokyo.ac.jp
}

\begin{abstract}
We propose a method of maintaining balance for a human-like character against large perturbations. The method enables a human-like model to maintain its balance with active whole-body motion, such as rotating its arms, bending down, and taking a step, if necessary. First, we capture the human motions of maintaining balance and abstract essential mechanisms from these motions. Next, we construct a model of maintaining balance that has a simple structure, such as an inverted pendulum. This model has two modes of maintaining balance: keeping the feet on the ground, and stepping. In this paper, the stepping mode is mainly described. Finally, we generate whole-body motion based on the model against several perturbations, and we discuss the validity of our method.
\end{abstract}

\section{INTRODUCTION}

Recently, interest in generating dynamically consistent motion of human-like characters has been increasing in various areas. In robotics, in order to operate a humanoid robot, it is necessary to generate motions that have strict dynamic consistency[1], [2]. In computer graphics, due to an increase in the demand for realistic three-dimensional animation, many researchers have developed techniques to generate consistent human motion easily[3], [4], [5].

When we consider realistic human motion, dynamic consistency of the motion is essential, and maintaining balance is fundamental to dynamic consistency. In a human-like model that supports its body by standing on two feet, the support area of the feet is small and the center of mass (CM) is in a high position; therefore, it is impossible to realize stable motion without an adequate method of maintaining balance. In particular, a method to cope with a sudden large perturbation is indispensable for stable motion.

When we consider maintaining balance of a human-like model, it is important to observe human strategy for maintaining balance, because humans themselves use flexible and effective ways to maintain balance. Thus, human strategy is a good guide to developing robust and effective systems. Humans employ large-scale whole-body active motions, such as bending down, rotating their arms, squatting down, taking a step, and so on. However, in research on maintaining balance of human-like models, such human techniques for maintaining balance have not been adequately studied.

With regard to previous work, some researchers have proposed methods to deal with large, unexpected perturbations. Zordan et al. designed a human model that receives joint torque as the input, and proposed a method to generate motion satisfying a space-time constraint and reacting to external perturbation, such as being boxed by someone [6]. However, a character was not allowed to take a step and it could only react passively. Fujiwara et al. developed a humanoid robot that can fall over safely, by reducing the impact of falling, and can stand up by itself [7]. In this work, an active reaction was generated to fall over safely and to stand up again. However, it is better that a character maintains its balance without falling down if possible.

In this study, we focus on such large-scale whole-body active motion against external perturbation and propose a method to generate it by using parameters observed from human motions. The basic concept of the proposed model of maintaining balance is as follows:

- The model is based on the observation of human motion.

- The model is made as simple a model as possible.

Regarding the first concept, we capture human motion using a motion-capturing system and force plates, and analyze it in order to extract essential characteristics from it. We then construct a model of maintaining balance based on it. Regarding the second concept, our model consists of a simple structure like an inverted pendulum model (IPM), the extracted parameters, and optimization calculation. It is controlled via global quantities, such as the $\mathrm{CM}$ and the zero moment point (ZMP). The advantages of a simple model are as follows:

- One can pay attention to only the essential part of motion.

- Effective whole-body motion can be generated even if the posture momentarily gets far away from a stable posture.

- Motion of maintaining balance can be decoupled from other motion.

In order to cope with larger perturbation, we design two modes for the model; maintaining balance by keeping feet on the ground and by stepping. In this paper, we mainly focus on the latter mode and describe it.

The contributions of this paper are that (1) we propose a model of maintaining balance using a simple structure whose parameters are extracted from human motions, and that (2) we show by simulation that characteristics of complex human whole-body motion for maintaining balance can be well represented using the simple model.

The remainder of the paper is organized as follows: Sec- 
tion II describes the model of maintaining balance. Section III shows simulation results of the model, and Section IV discusses them in aspects of appearance and dynamics. Finally, Section V concludes the paper.

\section{Model of Maintaining Balance}

In this paper, we propose a method to generate motion for maintaining balance against large perturbations applied to a human-like character. It consists of two modes. One is the method in which the character maintains its balance by keeping its feet planted firmly on the ground. The other is that in which the character takes a step to prevent itself from falling down.

When some sort of perturbation is applied to the character, it first tries to keep its balance by the mode of keeping its feet on the ground. Human-like motion, such as rotating arms and bending down, is generated here in order to maintain balance. In this mode, these motions are obtained as a result of optimization calculation. The detail of this method is described by our previous work [8].

However, because a character was not allowed to take a step in the first mode, it cannot maintain its balance when a perturbation is too large. Practically, we cannot find any solution in the optimization calculation. In such a case, the other mode, maintaining balance by stepping, is adopted. In the remainder of this paper, we describe this mode. Stepping motion is generated in the following way. First, trajectories of the $\mathrm{CM}$ and the feet are calculated by a simple physical model like an IPM. In order to determine control parameters for the model, we capture human motions using a motion-capturing system and force plates, analyze it, and extract essential parameters from it. Next, whole body motion is generated based on the trajectories of the CM by inverse kinematics (IK). Dynamic balance is not yet addressed in this step. Finally, using optimization calculation by the quadratic programming method, the motion is modified to a well-balanced motion. Motion is generated frame by frame; a generated posture of a frame is used as an initial posture of the next frame. We describe each step in the following sections.

\section{A. Determining Outline of Motion}

In order to generate a stepping motion, first, trajectories of the $\mathrm{CM}$ are determined by a physical model like an IPM. A stepping motion is modeled from two states. One is a state from the time when a swinging leg leaves the ground to when it again comes in contact with the ground. In this state, the model is supported by one leg. The other state is a state after the foot again contacts the ground, and the model is supported by both legs (Figure 1). In the former state, a rotational spring is assumed to be attached at the base of the IPM, and it generates torque to prevent the IPM from falling down. In the latter state, the stepping leg is assumed to be a spring that absorbs the shock of the foot contact.

We captured the human motions of maintaining balance by an optical motion-capturing system by Vicon Motion Systems

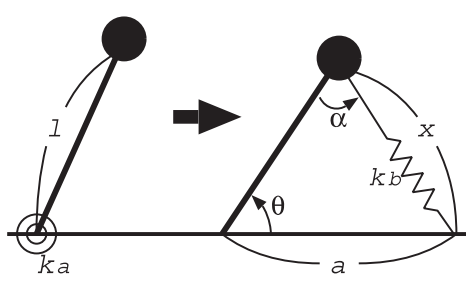

Fig. 1. Two states of IPM (before and after the foot contact): $\theta$ and $\alpha$ represent the lean angle and the stepping angle. $x$ and $a$ represent the length between the CM and the swing foot and the length between the support foot and the swing foot. These parameters at the foot contact are written as $\theta_{c}, \alpha_{c}$, $x_{c}$, and $a_{c}$. The spring constants of the springs are $k_{a}$ and $k_{b}$, respectively. The natural length of the latter spring is $x_{c}$.

with twelve force plates by KISTLER Japan. The motioncapturing system records the position of markers attached to a human body, and the force plates record the magnitude and the direction of the forces that act on the ground. Various strengths of force were applied near the CM of four subjects, who are male students. They were told to keep their balance, stepping only when necessary. The force was applied ten or fifteen times to each subject. They sometimes kept their balance without stepping, but at other times they had to take a step to prevent themselves from falling down.

First, we examine the spring constant $k_{a}$. In the phase before the foot contact, the IPM obeys the following equation:

$$
m l^{2} \ddot{\theta}=m l g \sin \theta-k_{a} \theta .
$$

Applying a human body to the IPM, $k_{a}$ is calculated based on the equation. The value is shown in Figure 3. According to the result, it can be said that the value of $k_{a}$ is small enough that we can consider $k_{a}$ is zero.

Next, we examine where the stepping foot contacts the ground. In order to determine this, it is necessary to determine $\alpha_{c}$, which is the stepping angle at the foot contact. According to observation of human motion, the ratio of $\alpha_{c}$ and $\theta_{c}$ is wellconverged independent of the subjects as shown in Figure 3. This figure shows the average and the standard deviation of $\alpha_{c} / \theta_{c}$ about each subject. Therefore, it can be said that $\alpha_{c} / \theta_{c}$ is always about 1.20 .

Finally, the spring constant $k_{b}$ is examined. According to our model, the relationship between $x$ and the ground reaction force acting on the contact foot $\left(f_{g}\right)$ follows the equation of $f_{g}=k_{b}\left(x-x_{c}\right)$. If the subjects actually moved obeying our model, points of $\left(f_{g}, x\right)$ lie on a straight line when we plot them on a two-dimensional graph. Figure 2 shows such a graph based on the captured data. We can see that the points definitely lie on a regression line. The spring constant, $k_{b}$, is obtained as the gradient of the regression line. The spring constant calculated from the captured data is shown as the table in Figure 3. Values of $k_{b}$ converge well about one subject, but the value is different among the subjects. This means that there is individual variation about $k_{b}$.

By using this IPM-like model with the parameters set by observing captured human motion, we construct the wholebody motion against large perturbations. 

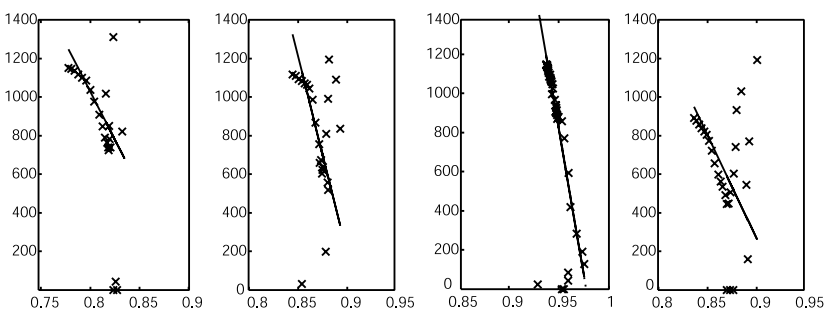

Fig. 2. Relationship between $x$ (distance between a foot contact point and $\mathrm{CM}$ ) and $f_{g}$ (the ground reaction force acting swing foot). Lines in the graphs are regression lines.

\begin{tabular}{|c||c|c||c|c||c|c|}
\hline \multirow{2}{*}{ Subject } & \multicolumn{2}{|c|}{$k_{a}$} & \multicolumn{2}{c|}{$\alpha_{c} / \theta_{c}$} & \multicolumn{2}{c|}{$k_{b}$} \\
\cline { 2 - 7 } & avg & std & avg & std & avg & std \\
\hline 1 & 79.9 & 184.4 & 1.29 & 0.215 & 25100 & 5400 \\
\hline 2 & 101.8 & 273.6 & 1.07 & 0.221 & 17900 & 7000 \\
\hline 3 & -83.5 & 560.7 & 1.30 & 0.167 & 12700 & 4200 \\
\hline 4 & -96.6 & 264.9 & 1.16 & 0.300 & 13500 & 4300 \\
\hline total & 15.8 & 356.3 & 1.20 & 0.256 & \multicolumn{2}{|c}{-} \\
\hline
\end{tabular}

Fig. 3. Parameters from captured data: The second, third, and fourth columns show parameters $k_{a}, \alpha_{c} / \theta_{c}, k_{b}$, respectively. With regard to $k_{b}$, the total is not calculated because individual variation is observed.

\section{B. Generating Lower Body Motion Using IK}

Next, lower body motion is generated based on the designed trajectories of the $\mathrm{CM}$ obtained in the previous section. IK calculation is used here. It is performed twice: IK for the CM is solved first, and then IK for the foot is solved. In the former, the link structure from the ankle of the support leg to the CM is considered. In the latter, the link structure from the hip joint of the swing leg to the heel is considered (Figure 4). The destination of the CM in solving IK is determined from the trajectory of the CM, which was calculated by the IPM. With regard to upper body motion, we consider it in the next section, and now the upper body posture in the previous frame is used as the upper body posture.

We solve IK by an iterative algorithm using a pseudoinverse matrix. Now, let $\boldsymbol{\theta}=\left(\theta_{0}, \ldots, \theta_{n}\right)^{T}$ be angles of the joints related to the link structure for considering IK structure, $\boldsymbol{p}_{i}$ and $\boldsymbol{z}_{i}$ be the position and the rotational axis of each Degree-of-freedom (DOF), and $\boldsymbol{r}=\left(\boldsymbol{P}^{T}, \boldsymbol{\Omega}^{T}\right)^{T}$ be the position and the rotation of the end effectors, such as the $\mathrm{CM}$ and the stepping foot. The angles of three DOF joints and the rotation are expressed by Euler angles. The relationship between $\boldsymbol{r}$ and $\boldsymbol{\theta}$ is written by Jacobian:

$$
J=\left(\begin{array}{cccc}
\boldsymbol{z}_{0} \times\left(\boldsymbol{P}-\boldsymbol{p}_{0}\right) & \cdots & \boldsymbol{z}_{i} \times\left(\boldsymbol{P}-\boldsymbol{p}_{i}\right) & \cdots \\
\boldsymbol{z}_{0} & \cdots & \boldsymbol{z}_{i} & \cdots
\end{array}\right) .
$$

The IK problem is solved using the pseudo-inverse matrix, $J^{T}\left(J J^{T}\right)^{-1}$, iteratively. The actual procedure of solving the IK is as shown in Figure 4.

\section{Generating Whole Body Motion by Optimization}

Finally, we generate whole body motion. Because only the lower body was considered in the previous section, there was redundancy in the upper body. In this section, well-balanced motion is generated by making use of the redundancy.

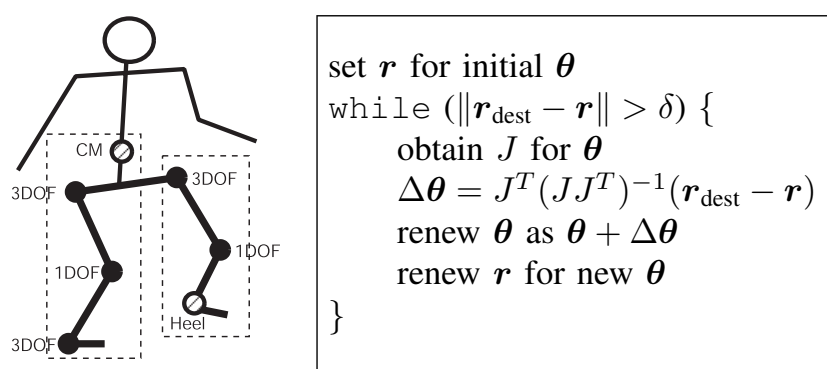

Fig. 4. Left: The link structures for solving IK. Two links are considered in this algorithm. Right: The procedure for solving IK.

Let $\boldsymbol{\theta}_{u}$ be the angles of the joints in the upper body. The problem in this section can be written as "obtaining angular acceleration $\ddot{\boldsymbol{\theta}}_{u}$ which satisfies the constraint that a ZMP should be within the foot support area." A ZMP is a concept proposed by Vukobratovic et al. [9] and is the point where a horizontal element of the moment induced by the ground reaction becomes zero. It is always within the support area if motion is dynamically consistent. The unknown variable in this problem is angular acceleration $\ddot{\boldsymbol{\theta}}_{u}$ because only it can be directly controlled by the system. Angles $\boldsymbol{\theta}_{u}$ and angular velocity $\dot{\boldsymbol{\theta}}_{u}$ are not directly controllable.

In order to solve the problem, the quadratic programming method is used. Because it allows only linear formulae as constraints, the constraint about a ZMP has to be expressed as a linear formula. From the definition of a ZMP, it is written as

$$
\boldsymbol{n}=(\boldsymbol{p}-\boldsymbol{s}) \times \boldsymbol{N},
$$

where $\boldsymbol{p}$ is the ZMP, $\boldsymbol{n}$ is the moment around the CM, $s$ is the position of the CM, and $N$ is the sum of the ground reaction force. Now let $\boldsymbol{p}$ be $\left(p_{x}, 0, p_{z}\right)^{T}$. Substituting the equation of motion, $\boldsymbol{N}=m(\ddot{\boldsymbol{s}}-\boldsymbol{g})$, the equation (3) can be solved as

$$
\begin{aligned}
& p_{x}=\frac{n_{z}+s_{x} m\left(\ddot{s}_{y}-g_{y}\right)-s_{y} m \ddot{s}_{x}}{m\left(\ddot{s}_{y}-g_{y}\right)} \\
& p_{z}=-\frac{n_{z}-s_{z} m\left(\ddot{s}_{y}-g_{y}\right)+s_{y} m \ddot{s}_{z}}{m\left(\ddot{s}_{y}-g_{y}\right)} .
\end{aligned}
$$

Here, because the position of the CM is a function of joint angles (i.e. $\boldsymbol{s}(\boldsymbol{\theta})$ ), and the angular momentum of the whole body is a function of joint angles and their velocity (i.e. $\boldsymbol{l}(\boldsymbol{\theta}, \ddot{\boldsymbol{\theta}}))$, both the acceleration of the CM $(\ddot{\boldsymbol{s}})$ and the moment acting around the CM $(\dot{l})$ can be written as linear functions about the acceleration of joint angles $(\ddot{\boldsymbol{\theta}})$ :

$$
\sum_{i} c_{i} \ddot{\theta}_{i}+d \text {. }
$$

Therefore, we can write a ZMP as a fraction of linear formulae about $\ddot{\theta}$ :

$$
p_{x}=\frac{\boldsymbol{c}_{x}^{T} \ddot{\boldsymbol{\theta}}+d_{x}}{\boldsymbol{c}_{c}^{T} \ddot{\boldsymbol{\theta}}+d_{c}}, \quad p_{z}=\frac{\boldsymbol{c}_{z}^{T} \ddot{\boldsymbol{\theta}}+d_{z}}{\boldsymbol{c}_{c}^{T} \ddot{\boldsymbol{\theta}}+d_{c}},
$$

where $\boldsymbol{c}_{*}$ and $d_{*}$ are constants. On the other hand, because the acceleration of the $\mathrm{CM}$ and the moment around the $\mathrm{CM}$ are 
calculated by dynamics calculation if angles, angular velocity, and angular acceleration of the joints are determined:

$$
\ddot{\boldsymbol{s}}=\ddot{\boldsymbol{s}}(\boldsymbol{\theta}, \dot{\boldsymbol{\theta}}, \ddot{\boldsymbol{\theta}}), \quad \ddot{\boldsymbol{n}}=\ddot{\boldsymbol{n}}(\boldsymbol{\theta}, \dot{\boldsymbol{\theta}}, \ddot{\boldsymbol{\theta}}) .
$$

the coefficients of the linear formulae in (6) are determined by calculating (8) about $\ddot{\boldsymbol{\theta}}^{i}$ for all $i$ :

$$
\ddot{\boldsymbol{\theta}}^{i}=(0, \ldots, 0, \stackrel{i \text {-th }}{\vee}
$$

Therefore, we can determine the coefficients in (7). If we approximate the foot support area by $N_{b}$ lines:

$$
D=\left\{\alpha_{i} x_{i}+\beta_{i} z_{i}+\gamma_{i}>0 \mid i=1, \ldots, N_{b}\right\},
$$

the constraint that a ZMP should be inside the foot support area is written as

$$
\alpha_{i} p_{x}+\beta_{i} p_{z}+\gamma_{i}>0 \quad\left(i=1, \ldots, N_{b}\right) .
$$

Substituting (7) in it, the condition can be written as linear inequalities about $\ddot{\boldsymbol{\theta}}$.

In addition to the constraint about a ZMP, we have to consider another constraint to prevent the character from jumping. If the denominator of (7) becomes negative, it means that the character is jumping. In this algorithm, we do not consider jumping motion, so the denominator should be always positive. This condition is written as $\ddot{s}_{y}>g$. This can be written as a linear formula because $\ddot{\theta}$ is written as a linear formula as shown above.

Satisfying these conditions, motion of the upper body is determined using optimization calculation. The quadratic programming method is used here. We take the following quadratic programming problem to determine the angular acceleration of the joints in the upper body.

$$
\begin{aligned}
\min & \ddot{\boldsymbol{\theta}}_{u}^{T} C \ddot{\boldsymbol{\theta}}_{u} \\
\text { s.t. } & \alpha_{i} p_{x}+\beta_{i} p_{z}>0 \quad\left(i=1, \ldots, N_{b}\right) \\
& \ddot{s}_{y}>g .
\end{aligned}
$$

The objective function aims to minimize the square summation of the angular acceleration. This is employed in order to generate low-cost motion. $C$ is a weight matrix. As mentioned above, the constraints can be written as linear inequalities about $\ddot{\boldsymbol{\theta}}$; therefore, they also can be written as linear inequalities about $\ddot{\boldsymbol{\theta}}_{u}$.

\section{Simulation Results}

In this section, results of simulation are shown when several types of perturbation are applied to the character. The generation of motion is performed every 0.01 second. Perturbation is applied to the CM of the model and control begins 0.2 second after perturbation is applied in order to simulate the response delay in a human case.

Figure 5 shows the result when a force of $300 \mathrm{~N}$ is applied for 0.1 second from the backward direction. The model maintains balance by rotating its arms. Humans also make these kinds of motions when they receive a sudden, large perturbation, because it is effective to reduce the effect of the induced angular momentum that makes them fall down forward.

Figure 6 shows the result when a force of $300 \mathrm{~N}$ is applied for 0.4 second from the backward direction. Compared with the previous experiment (Figure 5), the magnitude of the applied force is the same, but the duration is four times as long as in the first experiment. At first, the method by keeping the feet on the ground is employed, and the character rotates its arms to keep its balance. At last, however, it becomes impossible to maintain balance by using the method; then, maintaining balance by stepping is applied. Yellow bars in the figures stand for the inverted pendulum model used for generating steps.

We also apply the method to the situation where perturbation is applied during walking. The model of maintaining balance holds in the case of walking. However, a parameter of $\alpha_{c} / \theta_{c}$ is different. It is $\sim 1.4$ during walking while it is $\sim 1.2$ during standing upright. It means that a human takes a larger step when perturbation is applied during walking. Figure 7 shows the result. The first figure shows normal walking motion from the captured motion. At the time of the second figure, perturbation is applied to the model and our method begins to work. The velocity of the joints in the initial state is calculated from the captured data. A force of $300 \mathrm{~N}$ is applied for 0.4 second from the backward direction. After the perturbation, the character keeps stepping but the distance of stepping gets larger than normal walking and the waist is bent down.

\section{Discussion}

In this section, the results of simulation are compared with human motion. However, motions are different in every person, and, moreover, motions of the same person are different at different times. Therefore, it would not make sense to simply compare motions. In this study, we abstract essential parameters from captured human motion, make the model of maintaining balance based on the parameters, and reproduce whole-body motion from the behavior of the simplified model. If the characteristics of human motion, which are not modeled in the simplified model, appear in the reproduced motion, we may say that this is evidence that our model is reasonable.

\section{A. Apparent Aspect}

First, we compare the results of simulation with human motion in an aspect of appearance. In Figure 10, we examine two motions of maintaining balance: by keeping the feet on the ground and by stepping during walking. The upper rows show the captured human motions of maintaining balance when a force is applied. The middle rows illustrate characteristics of these motions. The lower rows show the results of simulation.

The figures on the left show the case of maintaining balance by keeping the feet on the ground. When a force is applied to a subject, who is a male, from his backward direction, his body leans forward and he begins to rotate his arms. It is effective in preventing humans from falling down because the momentum induced by this motion reduces the momentum of the whole body and prevents it from falling down. Next, he stretches 

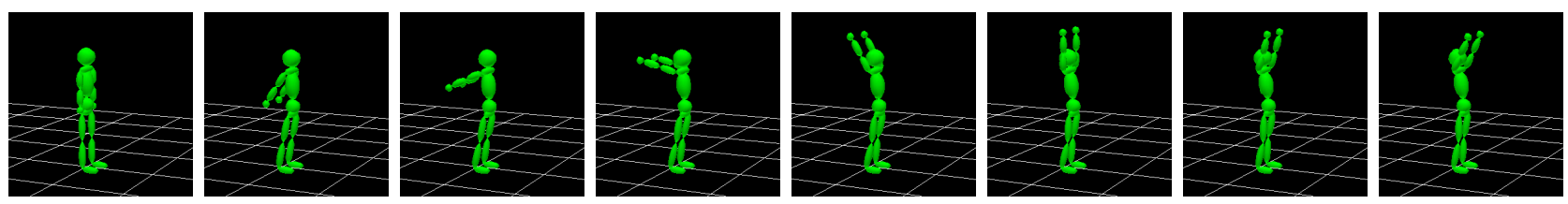

Fig. 5. Maintaining balance by keeping feet on the ground (300N, $0.1 \mathrm{sec}$, from backward direction)
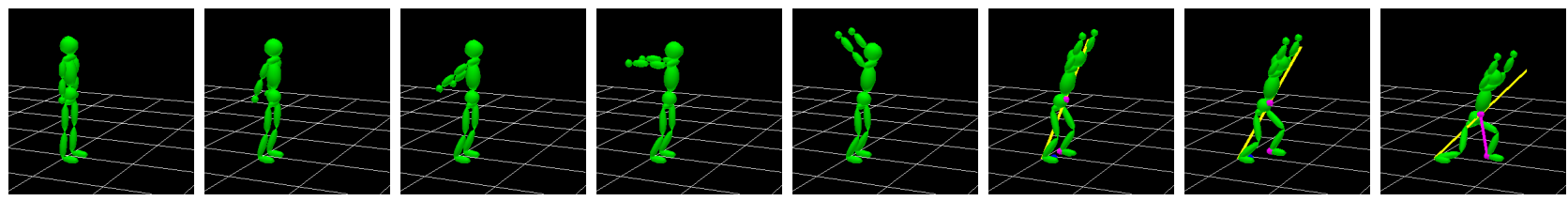

Fig. 6. Maintaining balance by stepping ( $300 \mathrm{~N}, 0.4 \mathrm{sec}$, from backward direction): First, the character maintains its balance by keeping the feet on the ground, but finally, it takes a step in order to prevent it from falling down.
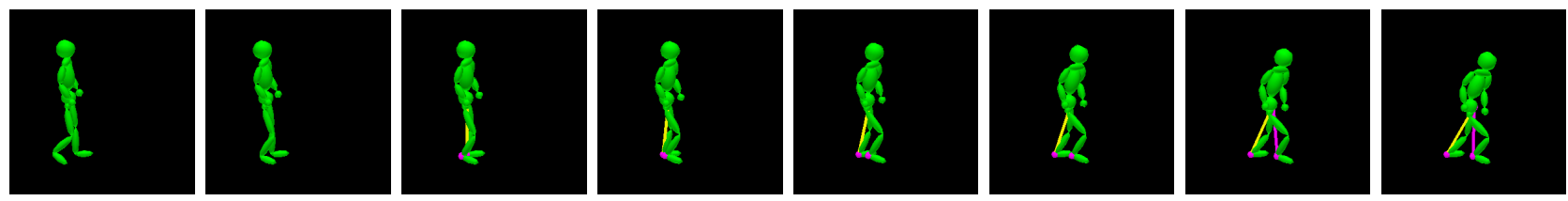

Fig. 7. Balance maintenance during walking $(300 \mathrm{~N}, 0.4 \mathrm{sec}$, from backward direction): The first frame shows captured walking motion. In the second frame, force is applied to the character, and then the method of maintaining balance by stepping is employed to generate the motion of maintaining balance.

his legs and bends down to move his waist backward, and then, he can recover his balance. We can see that the above characteristics are well represented in the generated motion.

The figures on the right show the case when perturbation is applied during walking. The force is applied from the backward direction when a human and a human-like character support their bodies by their left feet during walking. When perturbation is applied, the human takes a larger step to prevent himself from falling down. At the same time, he takes the counter action, moving his left shoulder and his left arm forward, to maintain his balance in a right-left direction. The characteristics of this human motion are also observed in the generated motion by simulation.

As shown above, the characteristics of human motion are well reproduced in the generated motion in both cases. An important point to emphasize is that only macro parameters, such as the CM and the ZMP, are considered in the model of maintaining balance; thus, such characteristics are not programmed in advance, but are rather the results of optimization calculation. The fact that the motion similar to a human is generated as the result of optimization calculation based on the simple model means that the model correctly represents the essential part of maintaining balance of a human.

\section{B. Dynamic Aspect}

Next, we compare results of simulation to human motion in an aspect of dynamics. Here, we focus on the magnitude of perturbation that can be handled without stepping. We performed an experiment in which perturbation was applied to a subject who was told to maintain his balance by keeping his feet on the ground as much as possible. A strain gauge was used then in order to record the applied force. The magnitude of the impulse that is applied in each trial is calculated by integrating the output of the strain gauge. Figure 8 shows the calculated impulse and whether the subject could maintain balance without stepping or not.

According to the table, we can estimate the limit of the impulse for which the subject could maintain balance without stepping. For perturbation from the backward direction, the limit is estimated as $\sim 40 \mathrm{Ns}$, and for perturbation from the forward direction, it is estimated as $\sim 35$ Ns. In the latter case, the subject could maintain balance without stepping in the fourth trial although the larger perturbation of $44.5 \mathrm{Ns}$ was applied. However, the subject's success in maintaining balance occurred mainly because the subject by mistake made a counter motion before perturbation was applied.

Comparing this, the proposed model generates stable motion against perturbation from backward and forward directions for the perturbation of $\sim 35 \mathrm{Ns}$ and $\sim 30 \mathrm{Ns}$ respectively. If the perturbation is increased, the result becomes unstable. For an impulse of $40 \mathrm{Ns}$ from the backward direction, the method could not find a solution. For an impulse of $35 \mathrm{Ns}$ from the forward direction, the method also could not find the solution.

Figure 9 is a graph to compare the result of simulation with the human case. The upper band represents the case when perturbation is applied from a backward direction, and the lower band represents the case when perturbation is applied from a forward direction. The horizontal axis represents the applied impulse. The green area shows the range of impulse in which a solution of the optimization calculation is stably found, while the red area shows the range of impulse in which no solution is found without stepping. The mark ' $O$ ' means that the subject could maintain his balance without stepping 


\begin{tabular}{ccc}
\hline Trial & Impulse & Step \\
\hline 1 & 29.4 & no step \\
2 & 35.9 & no step \\
3 & 54.7 & step \\
4 & 43.5 & step \\
5 & 38.4 & no step \\
\hline
\end{tabular}

\begin{tabular}{ccc}
\hline Trial & Impulse & Step \\
\hline 1 & 29.1 & no step \\
2 & 39.0 & step \\
3 & 34.0 & no step \\
4 & 44.5 & no step \\
5 & 47.8 & step \\
6 & 36.7 & step
\end{tabular}

Fig. 8. Impulse applied to the subject: The tables show the magnitude of the impulse (Ns) applied to the subjects when the force applied from the backward direction (the left) and from the forward direction (the right). The column "Step" shows whether the subject could maintain balance without stepping or not. "no step" means he could and "step" means he could not.

for the impulse, while the mark ' $x$ ' means that he could not. According to the graph, it can be said that the human character under the proposed method shows a similar dynamic property to a human regarding the limitation of impulse that can be handled without stepping.

In the proposed model, the mode by keeping the feet on the ground is taken at first, and if perturbation is too large, it switches to the mode by stepping. The largeness of perturbation is judged by whether any solution is found or not at the optimization step in the mode by keeping the feet on the ground. Therefore, the above correspondence between simulation results and human motion shows that the threshold to choose the mode can be well represented by our model.

\section{CONCLUSiON}

In this paper, we discuss motion for maintaining balance against perturbation, in particular sudden and large perturbation, and propose a method to generate such motion using a simple mechanism based on observation of human motion.

First, we capture human motion using a motion-capturing system and force plates, and abstract essential parameters controlling the motion. Next, we make a model for maintaining balance based on the parameters. Finally, we generate the whole-body motion of maintaining balance by optimization calculation against several kinds of perturbation. In practice, we model two types of whole-body motion of maintaining balance: keeping the feet on the ground, and stepping. In this paper, we mainly describe the latter mode.

Comparing the generated motion by the model with human motion, it is found that characteristics of human motion are well reproduced in the generated motion. In addition, we examine the magnitude of perturbation that can be handled without stepping. As a result, a good correspondence appears between the generated motion and human motion. It shows the fact that the generated motion represents characteristics of human motion not only in an apparent aspect but also in a quantitative aspect.

\section{ACKNOWLEDGMENT}

The authors would like to thank Prof. Minoru Ueda for his support in enabling us to use the motion-capturing system in Aizu University.

This work is supported in part by the Japan Science and Technology Corporation (JST) under the CREST project, and in part by the Grant-in-Aid for Scientific Research on Priority Areas (c)

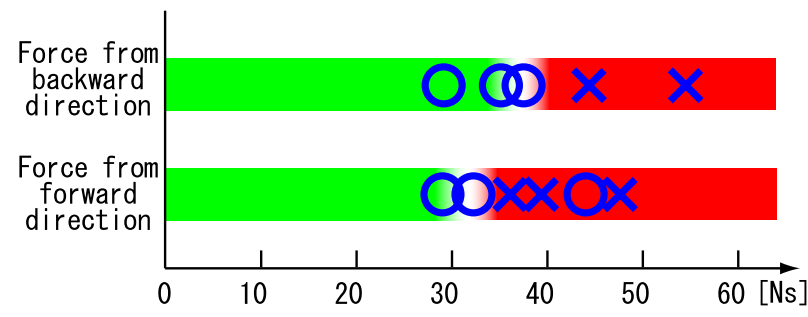

Fig. 9. Comparison of impulse that can be handled without stepping: The horizontal axis represents applied impulse. The green/red area shows the range of impulse in which the optimization problem can/cannot be solved. The mark $\bigcirc / \times$ means that the subject could/could not maintain his balance without stepping.
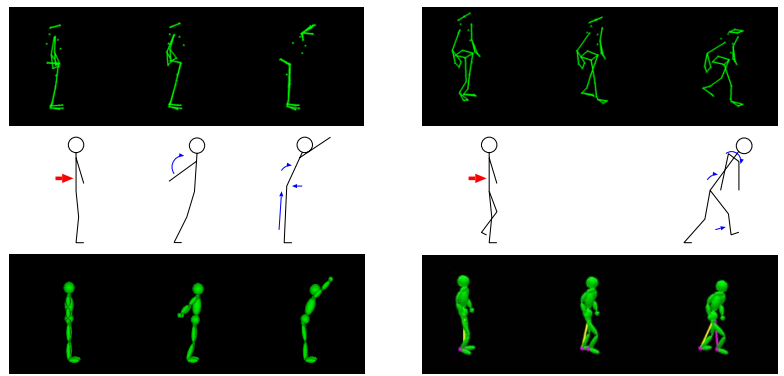

Fig. 10. Human motion vs. generated motion: The figures on the left show the case of maintaining balance by keeping feet on the ground, while the figures on the right show the case of maintaining balance during walking. The upper rows show the captured human motion, the middle rows show their characteristics, and the lower rows show the simulation results.

16016218 of the Ministry of Education, Culture, Sports, Science and Technology.

\section{REFERENCES}

[1] K. Nishiwaki, S. Kagami, Y. Kuniyoshi, M. Inaba, and H. Inoue, "Online generation of humanoid walking motion based on a fast generation method of motion pattern that follows desired zmp," in Proceedings of the IEEE/RSJ International Conference on Intelligent Robots and Systems (IROS), 2002, pp. 2684-2689.

[2] S. Kajita, F. Kanehiro, K. Kaneko, K. Jujiwara, K. Harada, K. Yokoi, and H. Hirukawa, "Resolved momentum control: Humanoid motion planning based on the linear and angular momentum," in Proceedings of the 2004 IEEE/RSJ International Conference on Intelligent Robots and Systems (IROS), 2004, pp. 1644-1650.

[3] S. Tak, O. Song, and H. Ko, "Motion balance filtering," Computer Graphics Forum, vol. 19, no. 3, pp. 435-446, 2000.

[4] C. K. Liu and Z. Popvić, "Synthesis of complex dynamic character motion from simple animations," ACM Transactions on Graphics (SIGGRAPH 2002), vol. 21, no. 3, pp. 408-416, 2002.

[5] A. C. Fang and N. S. Pollard, "Efficient synthesis of valid human motion," ACM Transactions on Graphics (SIGGRAPH 2003), vol. 22, no. 3, pp. 417-426, 2003

[6] V. B. Zordan and J. K. Hodgins, "Motion capture-driven simulations that hit and react," in ACM SIGGRAPH Symposium on Computer Animation, 2002.

[7] K. Fujiwara, F. Kanehiro, S. Kajita, K. Yokoi, H. Saito, K. H. nad Kenji Kaneko, and H. Hirukawa, "The first human-size humanoid that can fall over safely and stand-up again," in Proceedings of the IEEE/RSJ International Conference on Intelligent Robots and Systems (IROS), 2003, pp. 1920-1926.

[8] S. Kudoh, T. Komura, and K. Ikeuchi, "The dynamic postural adjustment with the quadratic programming method," in In Proceedings of IEEE/RSJ International Conference on Intelligent Robots and Systems, 2002, pp. 2563-2568.

[9] M. Vukobratović and J. Stepanenko, "On the stability of anthropomorphic systems," Mathematical Biosciences, vol. 15, pp. 1-37, 1972. 